\title{
Translation, cultural adaptation, and validation of the American Skindex-29 quality of life index ${ }^{*}$
}

\author{
Henrique Ribeiro de Paula ${ }^{1}$ \\ Gal Moreira Dini ${ }^{1,2}$
}

\author{
Alessandra Haddad ${ }^{1}$ \\ Lydia Masako Ferreira $^{1}$
}

Mariana Alves Weiss ${ }^{1}$

Abstract: BACKGROUND: Measuring the quality of life measure of patients with dermatologic diseases is an important concern. The instruments to evaluate it are commonly originally written in English and need to be translated and validated to be used in different cultures.

Oвjective: The purpose of this paper is to translate and validate the Skindex-29 questionnaire to Brazilian Portuguese to be used in our country as a quality of life assessment instrument in dermatologic patients.

METHODS: The first step was the translation from English to Brazilian Portuguese and the back-translation by two native speakers. The translated version was then used for the second step, when three questionnaires were applied to 75 patients ( 43 of whom were classified as lightly affected and 32 as heavily affected by their dermatologic conditions): an identification questionnaire, the translated version of Skindex-29, and the Brazilian Portuguese version of Dermatologic Life Quality Index (DLQI). Additionally, the generic questionnaire Short Form 36 (SF-36) was applied to 41 of these patients. The last step to evaluate reproducibility was repeating the Skindex-29 questionnaire by the same researcher one week later in 44 patients.

RESULTS: Reliability was observed in global Skindex-29 scale $(\alpha=0.934)$, and its domains emotions $(\alpha=0.926)$, symptoms $(\alpha=0,702)$, and psycosocial functioning $(\alpha=0.860)$. The reproducibility showed high intraclass correlations. High intra class correlations was observed, thus validating reliability.

CONCLUSIONS: The Skindex-29 quality of life questionnaire was properly translated and validated to Brazilian Portuguese.

Keywords: Dermatology; Quality of life; Questionnaires; Translations; Validation studies

\section{INTRODUCTION}

Measuring the quality of life of patients with dermatologic diseases is an important and especially challenging concern..$^{1,2}$ The most commonly used instruments to assess quality of life were originally written in English and need to be translated and adapted for use in other cultures. ${ }^{3,45}$ Many questionnaires to evaluate quality of life were designed specifically for patients with skin conditions. ${ }^{6,7}$ Disease-specific instruments are preferable to generic health-related quality of life questionnaires in certain patient populations, because they are more sensitive to the areas most affected by the disease. ${ }^{8}$

Culturally adapting an instrument has many advantages over writing a new one. For example, it reduces the costs and the time spent in the writing process and allows for using the instrument, which has been widely used before, to make intercultural comparisons. ${ }^{9}$ It should also be considered that an adapted questionnaire can be used both for native speaker patients and for immigrant patients, thus avoiding selection bias and improving its measuring skills.

In dermatology, studies have shown that health-related quality of life may be severely reduced in patients with a wide range of skin diseases such as atopic dermatitis, psoriasis, leprosy, acne vulgaris, skin tumors, onychomycosis, and skin ulcers. ${ }^{10-19} \mathrm{On}$ the other hand, studies in plastic surgery have analyzed changes in quality of life caused by surgical procedures, focusing on breast, nasal, facial and abdominal procedures..$^{20-25}$

Daily life domains that may be affected by skin disease include aspects related to physical and emotional well-being, which may have an impact on social life and productivity at work and school. The effects of skin diseases on social interactions and emotional patterns may be especially relevant, because the 
symptoms of these diseases usually affect patient's appearance. Skin disease often has a profound impact on patient's life, and medical judgment is not well correlated with the perception of affected individuals on disease severity. ${ }^{26}$ Thus, measuring the subjective impact of the disease on the patient becomes particularly important.

The Skindex-29 is a disease-specific questionnaire that comprehensively assesses the effects of skin diseases on patient's quality of life. ${ }^{27}$ It was specifically developed to detect changes throughout the time, as well as differences among patients with different skin diseases. The questionnaire covers areas considered crucial in an instrument designed to evaluate quality of life, such as: degree of symptoms, psychosocial functioning, and emotional status. ${ }^{28,29}$ The 29 -item version is a refinement of a previous 61 -item version ${ }^{8,27}$ and combines the advantage of an easier administration with improved psychometric properties. It was originally written in English and has already been translated and validated to other languages: Spanish, Italian, German, French, Turkish, among others. ${ }^{26,30,31,32}$

The aim of the present study was to translate the Skindex-29 questionnaire to Brazilian Portuguese and investigate its measuring properties, in order to allow its use as a quality of life assessment instrument in patients with dermatologic diseases in Brazil.

\section{METHOD}

A preliminary authorization to translate and validate the questionnaire was obtained from its original authors.

In order to develop a valid Brazilian Portuguese version of the Skindex-29, the study followed some guidelines for the cultural adaptation of health-related quality of life instruments.

The translation phase involved six stages:

1. Translation of the Skindex-29 into Portuguese by two English teachers who were Portuguese native speakers.

2. Development of a single Brazilian Portuguese version by consensus.

3. Back-translation of the first Brazilian Portuguese version by two American translators unaware of the study purposes. Comparison of the backtranslated version with the original English language instrument. Possible discrepancies were documented and analyzed by a multidisciplinary committee with regard to their applicability and cultural equivalence.

4. Evaluation by a multidisciplinary committee: the committee evaluated both original and translated versions, as well as filling instructions and scales. If needed, sentences were rewritten and scales adjusted until obtaining the second Brazilian Portuguese version. The committee's analysis involved:

a. Resolution of discrepancies: modifications in instructions or in the questionnaire format, modification or rejection of inappropriate items, and generation of new items.

b.Adjustment of scales: semantic equivalence, idiomatic equivalence, practical equivalence, and conceptual equivalence.

5. Pretest of an intermediate Brazilian Portuguese version in a pilot group of individuals with and without skin diseases to determine if the translated version was understandable. In this process, subjects made comments on the meaning, interpretation and any potential ambiguity for each item.

6. Translation and back-translation of any problematic item found in the pretest for the generation of the final Brazilian Portuguese version of the instrument.

\section{Preliminary evaluation of measuring properties Specimens}

An identification questionnaire, the final Brazilian Portuguese version of the Skindex-29, and the Brazilian Portuguese version of the Dermatology Life Quality Index (DLQI) questionnaire were applied to 75 adults selected by convenience. Of the total number of patients, 43 came from the Cosmetolgy outpatient clinic from Casa de Cirurgia Plástica of Universidade Federal de São Paulo and did not show facial lesion on the days of data collection(plastic surgery group), 32 came from the Dermatology outpatient clinic of the Universidade Federal de São Paulo, presented with inflammatory dermatoses such as psoriasis or atopic dermatitis and had a facial lesion larger than $3 \mathrm{~cm}$ on the days of data collection (dermatology group). These outpatient clinics were selected because they had a good number of target patients for this research.

In order to add one more quality of life questionnaire for comparison, the last 43 patients of both clinics answered the Short Form 36 (SF-36) questionnaire. After one week, the Skindex-29 was readministered by the same evaluator to patients who agreed to return to the clinic.

The questionnaires were administered during the month of August, 2011 on the corresponding days of each outpatient clinic.

\section{Instruments}

For the present validation study, two other quality of life assessment questionnaires were used along with the Skindex-29: the DLQI and the SF-36. 
The Skindex-29, a refined version of a previous 61-item questionnaire, is a questionnaire with 29 selfadministered questions that assess the health-related quality of life of patients with skin diseases. It evaluates three domains: degree of symptoms, psychosocial functioning, and emotional status. The possible answers are: never, rarely, sometimes, often, and always, given in a scale from 1 to 5 points respectively, and the final scale score is established either by the mean for the points obtained in the 29 questions (total score) or by the mean for the items of each domain (domain score).

The DLQI is a brief 10-item health-related quality of life questionnaire specific for dermatology. ${ }^{33}$ This is a well-established instrument validated in Brazil that shows good psychometric properties and provides a total score. ${ }^{5}$

The SF-36 is a generic quality of life assessment instrument that has already been validated in Brazil and is easy to administer and understand. It is a multidimensional questionnaire formed by 36 items covering eight scales or components: physical functioning, physical role functioning, bodily pain, general health, vitality, social functioning, emotional role functioning, and mental health. In addition, it yields a final score ranging from 0 to 100, in which zero (0) corresponds to the worst general health status and 100 to the best general health status. ${ }^{3,34}$

\section{Statistics}

Categorical variables were expressed as absolute and relative frequencies. The association between two categorical variables were analyzed using the chi-square test or, alternatively, the Fisher's exact test in the case of small samples. On the other hand, the means of patients from plastic surgery and dermatology groups were compared using the Student $t$ test for independent samples or the nonparametric Mann-Whitney test in case of violation of normality assumption.

The internal consistency between the items that comprise the Skindex-29 dimensions was expressed as Cronbach's alpha coefficients.

The capacity of the Skindex-29 to investigate patients' quality of life in terms of skin (validation) was evaluated by comparing mean total scores and mean scores for the different instrument dimensions (emotional, symptomatic and functional aspects) between plastic surgery and dermatology patients. In addition, this study aimed to assess the extent to which Skindex-29 scores are associated with other already known and validated scores, such as those obtained from DLQI and SF-36 questionnaires, using Spearman's correlations.

Variable normal distribution was tested using the Kolmogorov-Smirnov test, and exploratory data analysis focused on obliquity (skewness), which measures the asymmetry of distribution tails, and the kur- tosis measure, which estimates the degree of flattening and narrowing of the distribution curve. In these analyses, approximately normal distribution was not rejected.

Intraobserver reproducibility was assessed by intraclass correlation coefficient and by the Student's $t$ test comparing mean scores between the two times when questionnaires were administered.

\section{RESULTS}

\section{First phase: translation}

The analysis from the multidisciplinary group did not show discrepancies between original and back-translated versions. There was no need of modifying questionnaire instructions or format, modifying or rejecting inappropriate items, nor generating new items with significant discrepancies. Thus, no change in the first translation of the instrument was required, and the research entered the second phase.

The pretest, administered to 11 patients, did not find problems of meaning, interpretation and potential ambiguity, which made it possible to develop the final version of the Skindex-29 translated into Brazilian Portuguese (Chart 1).

\section{Second phase: evaluation of measuring properties}

According to table 1, patients from both groups showed a similar distribution in terms of gender and educational level. Thus, in general, most subjects were female $(76.0 \%)$ and had an educational level of up to high school education (76.1\%). Patients' mean age was 47.8 years (standard deviation $=17.8$ ), with a minimum age of 18 years and a maximum age of 91 years. Both groups showed similar mean ages $(\mathrm{p}=0.342)$.

\section{Assessment of internal consistency of the Skindex-29}

According to table 2, global Skindex-29 score had excellent reliability (Cronbach's alpha $=0.934$ ), as well as its dimensions related to emotions (Cronbach's alpha $=0.926)$, symptoms, and psychosocial functioning. No significant increase was observed in total and inter-dimensional Cronbach's alpha with the removal of any item from the questionnaire.

\section{Reproducibility assessment}

Table 3 describes mean global Skindex-29 scores and mean scores for each Skindex-29 subscale at each time of assessment by the same evaluator in 44 patients. According to this table, extremely high intraclass correlations were observed, which points out to a good reproducibility. Corroborating this fact, a strong correlation could be observed between the scores obtained in the two measuring times, with no difference between means (Graph 1). 
CHART 1: Translation of the Skindex-29 into Portuguese

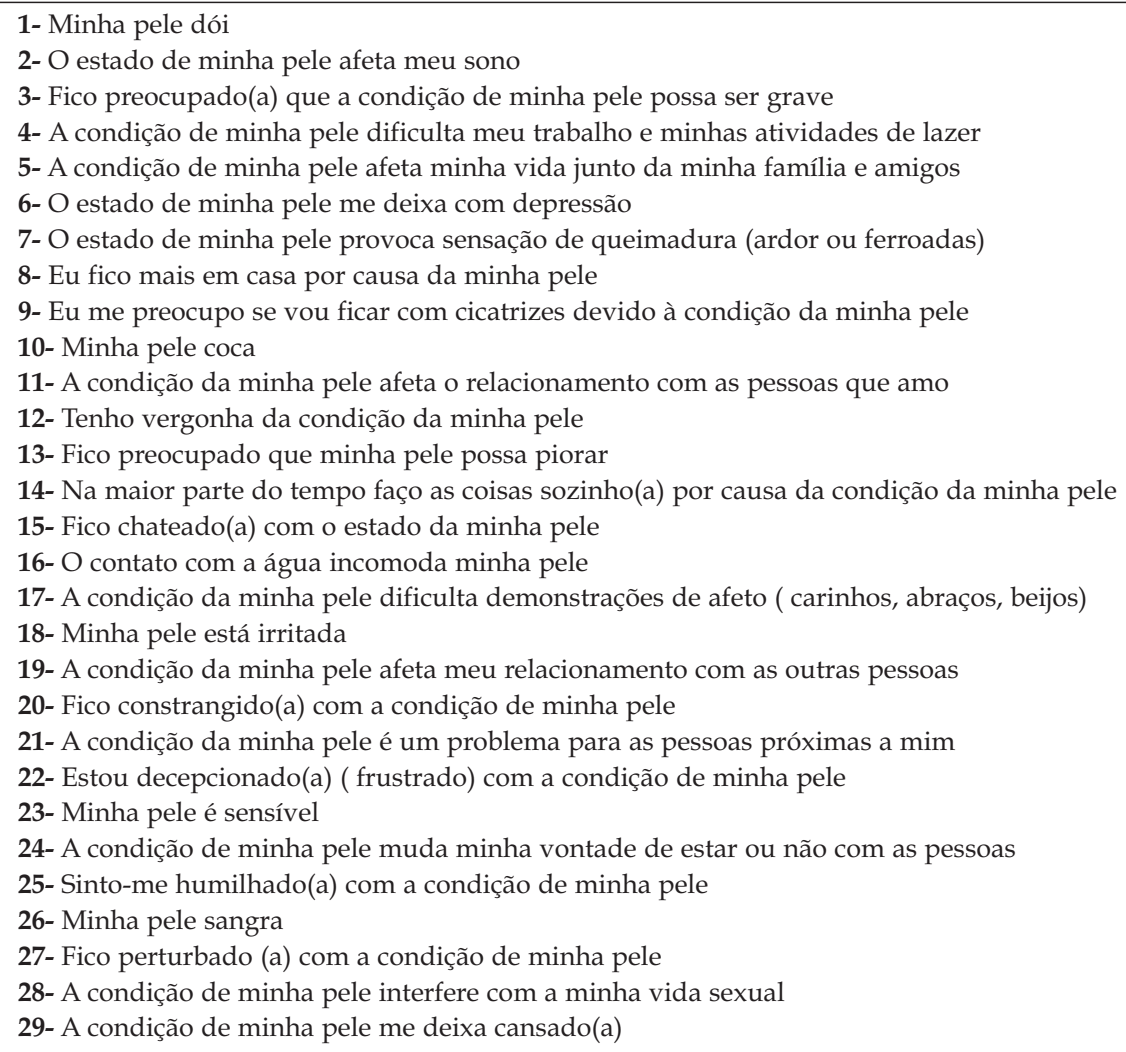

Para todas as frases as respostas possíveis são: nunca, raramente, às vezes, frequentemente e o tempo todo Source adapted: Chren MM, et al. 1996.7

TABLE 1: Distribution of patients' characteristics, according to groups

\begin{tabular}{|c|c|c|c|c|c|c|}
\hline \multirow[t]{3}{*}{ Characteristics } & \multicolumn{4}{|c|}{ Group } & \multicolumn{2}{|c|}{ Total } \\
\hline & \multicolumn{2}{|c|}{ Dermatology } & \multicolumn{2}{|c|}{ Plastic surgery } & \multirow[b]{2}{*}{$\mathbf{N}$} & \multirow[b]{2}{*}{$\%$} \\
\hline & $\mathbf{N}$ & $\%$ & $\mathbf{N}$ & $\%$ & & \\
\hline Gender & 32 & $100.00 \%$ & 43 & $100.00 \%$ & 75 & $100.00 \%$ \\
\hline Female & 24 & $75.00 \%$ & 33 & $76.70 \%$ & 57 & $76.00 \%$ \\
\hline Male & 8 & $25.00 \%$ & 10 & $23.30 \%$ & 18 & $24.00 \%$ \\
\hline \multicolumn{7}{|l|}{$x^{2}=0.03(p=0.861)$} \\
\hline Educational level & 28 & $100.00 \%$ & 39 & $100.00 \%$ & 67 & $100.00 \%$ \\
\hline Elementary school & 15 & $53.60 \%$ & 13 & $33.30 \%$ & 28 & $41.80 \%$ \\
\hline High school & 7 & $25.00 \%$ & 16 & $41.00 \%$ & 23 & $34.30 \%$ \\
\hline College education & 6 & $21.40 \%$ & 10 & $25.60 \%$ & 16 & $23.90 \%$ \\
\hline No data & & & & & 8 & \\
\hline$x^{2}=2.94(p=0.230)$ & & & & & & \\
\hline
\end{tabular}


TABLE 2: Summary of Skindex-29 measures (Cronbach's alpha and Spearman's coefficient)

\begin{tabular}{|c|c|c|c|c|c|}
\hline & Skindex & $\begin{array}{l}\text { Mean } \\
\text { deviation }\end{array}$ & $\begin{array}{l}\text { Standard } \\
\text { an item is excluded }\end{array}$ & $\begin{array}{l}\text { Alpha if } \\
\text { with score }\end{array}$ & Spearman's coefficient \\
\hline \multirow{10}{*}{$\begin{array}{l}\text { Emotions } \\
(\text { Alpha }=0.926)\end{array}$} & Item 3 & 2.27 & 1.47 & 0.925 & $0.696^{* *}$ \\
\hline & Item 6 & 1.83 & 1.16 & 0.92 & $0.725^{\star *}$ \\
\hline & Item 9 & 2.28 & 1.58 & 0.927 & $0.645^{\star *}$ \\
\hline & Item 12 & 2.29 & 1.52 & 0.912 & $0.834^{* *}$ \\
\hline & Item 13 & 2.44 & 1.6 & 0.919 & $0.758^{* *}$ \\
\hline & Item 15 & 2.37 & 1.5 & 0.909 & $0.882^{* *}$ \\
\hline & Item 20 & 2.17 & 1.41 & 0.914 & $0.817^{* *}$ \\
\hline & Item 22 & 2.23 & 1.55 & 0.914 & $0.794^{* *}$ \\
\hline & Item 25 & 1.47 & 1.08 & 0.926 & $0.561^{* *}$ \\
\hline & Item 27 & 1.83 & 1.28 & 0.914 & $0.779^{* *}$ \\
\hline \multirow{7}{*}{$\begin{array}{l}\text { Symptoms } \\
(\text { Alpha = 0.702) }\end{array}$} & Item 1 & 1.27 & 0.78 & 0.689 & $0.394^{* *}$ \\
\hline & Item 7 & 1.81 & 1.16 & 0.654 & $0.629 * *$ \\
\hline & Item 10 & 2.28 & 1.32 & 0.6 & $0.763^{* *}$ \\
\hline & Item 16 & 1.12 & 0.46 & 0.699 & $0.263^{*}$ \\
\hline & Item 18 & 2.08 & 1.19 & 0.618 & $0.780^{* *}$ \\
\hline & Item 23 & 2.85 & 1.57 & 0.739 & $0.626^{* *}$ \\
\hline & Item 26 & 1.28 & 0.71 & 0.664 & $0.482^{* *}$ \\
\hline \multirow{12}{*}{$\begin{array}{l}\text { Psychosocial functioning } \\
(\text { Alpha }=0.860)\end{array}$} & Item 2 & 1.47 & 1.03 & 0.855 & $0.519^{* *}$ \\
\hline & Item 4 & 1.97 & 1.53 & 0.847 & $0.705^{* *}$ \\
\hline & Item 5 & 1.47 & 1 & 0.858 & $0.490^{* *}$ \\
\hline & Item 8 & 1.57 & 1.2 & 0.855 & $0.627^{* *}$ \\
\hline & Item 11 & 1.44 & 1.07 & 0.85 & $0.550^{* *}$ \\
\hline & Item 14 & 1.49 & 1.18 & 0.85 & $0.580^{* *}$ \\
\hline & Item 17 & 1.55 & 1.2 & 0.837 & $0.662^{* *}$ \\
\hline & Item 19 & 1.59 & 1.15 & 0.835 & $0.665^{* *}$ \\
\hline & Item 21 & 1.2 & 0.68 & 0.854 & $0.404^{* *}$ \\
\hline & Item 24 & 1.57 & 1.16 & 0.834 & $0.684^{* *}$ \\
\hline & Item 28 & 1.4 & 1.04 & 0.851 & $0.505^{* *}$ \\
\hline & Item 29 & 1.65 & 1.24 & 0.856 & $0.579 * *$ \\
\hline
\end{tabular}

Overall

(Alpha $=0.934)$

${ }^{*} \mathrm{p}<0.05 .{ }^{*} \mathrm{p}<0.01$

TABle 3: Mean, Standard Deviation, Spearman's Correlation, and Intraclass Correlation

\begin{tabular}{|c|c|c|c|c|c|}
\hline & \multicolumn{2}{|c|}{ Score (mean \pm standard deviation) } & \multirow[t]{2}{*}{ Spearman's correlation } & \multirow{2}{*}{ Intraclass correlation } & \multirow[t]{2}{*}{$\mathbf{N}$} \\
\hline & Before & After & & & \\
\hline Emotions & $2.51 \pm 1.08$ & $2.49 \pm 1.12$ & $0.922^{* *}$ & $0.933^{* *}$ & 44 \\
\hline Symptoms & $1.96 \pm 0.67$ & $2.03 \pm 0.67$ & $0.908^{* *}$ & $0.932 * *$ & 43 \\
\hline Psychosocial functioning & $1.72 \pm 0.73$ & $1.74 \pm 0.74$ & $0.906^{* *}$ & $0.899 * *$ & 44 \\
\hline Total Skindex-29 score & $2.06 \pm 0.69$ & $2.08 \pm 0.70$ & $0.926^{\star *}$ & $0.937^{* *}$ & 43 \\
\hline \multicolumn{6}{|c|}{ One patient did not report any information in one of the items from the Symptoms dimension. } \\
\hline \multicolumn{6}{|c|}{ Emotions: $\mathrm{t}=0.298(\mathrm{p}=0.767)$} \\
\hline \multicolumn{6}{|c|}{ Symptoms: $\mathrm{t}=1.710(\mathrm{p}=0.095)$} \\
\hline \multicolumn{6}{|c|}{ Psychosocial functioning: $\mathrm{t}=0.455(\mathrm{p}=0.652)$} \\
\hline \multicolumn{6}{|c|}{ Total Skindex score: $\mathrm{t}=0.510(\mathrm{p}=0.613)$} \\
\hline
\end{tabular}




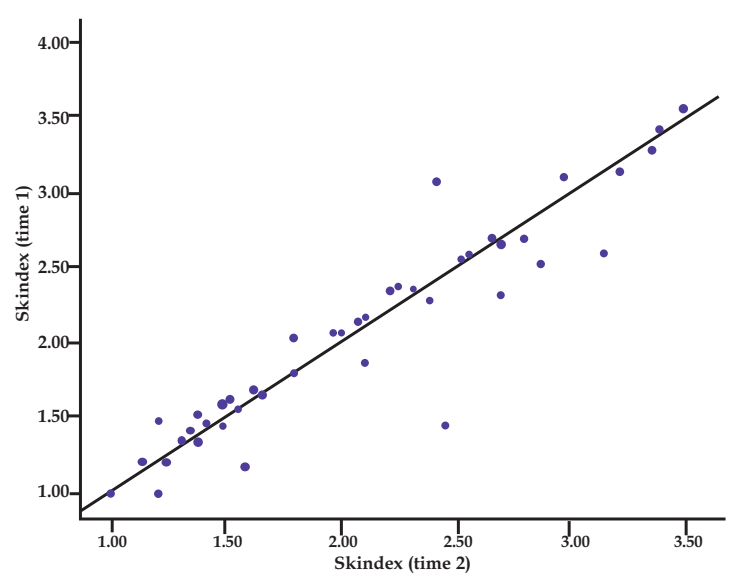

GraPH 1: Dispersion graph of total Skindex score (gross score) in the two evaluations. Dispersion

\section{Scale validation}

Graph 2 shows a higher score variability (interquartile range) in the dermatology group than in the cosmetology group, in addition to the presence of points outside the curve in the subscales associated with symptomatic and functional dimensions in the dermatology group and in emotional, functional and overall dimensions in the cosmetology group. Graph 3 demonstrates the $95 \%$ confidence intervals for mean values of Skindex scales.

In graph 4, it was possible to observe that, both for all Skindex-29 domains and for global Skindex-29 score, mean values from the patients heavily affected by skin diseases (plastic surgery group) were higher than those from the lightly affected patients (cosmetology group), revealing that, as expected, the perception of quality of life related to skin was better in lightly affected patients than in heavily affected patients.

According to table 4, the correlation between Skindex-29 and DLQI was higher than that between Skindex-29 and SF-36.

\section{DISCUSSION}

The present evaluation of the measuring properties of the Brazilian Portuguese version of the Skindex-29 suggests that this questionnaire is a reliable and valid method to quantify the quality of life of Brazilian patients with skin diseases. The strategy of translating the original version of the questionnaire into Brazilian Portuguese, performing a back-translation into English, and then conducting a pretest followed the universally accepted steps for the development of a conceptually and linguistically equivalent translation of quality of life questionnaires. ${ }^{8,935}$ Pretesting, including interviews with individuals from the community and patients, is especially impor-

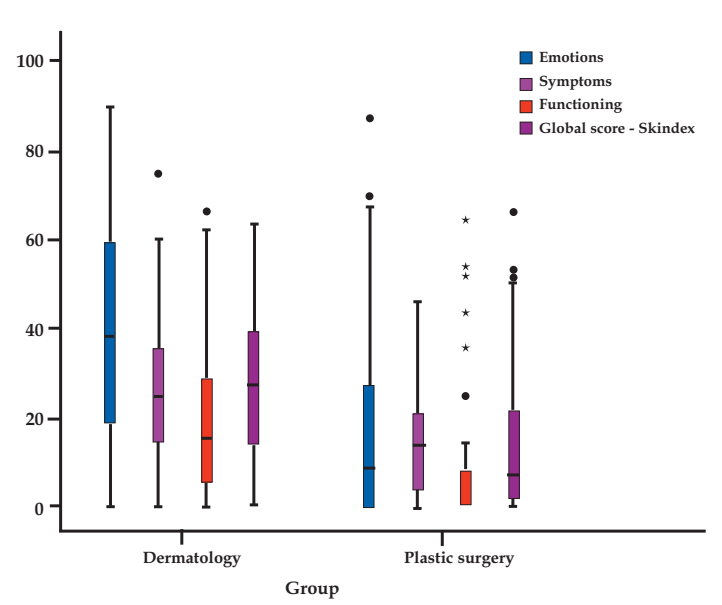

Graph 2: Box-Plot of subscales and global Skindex-29 scores per group. Global and subscale scores

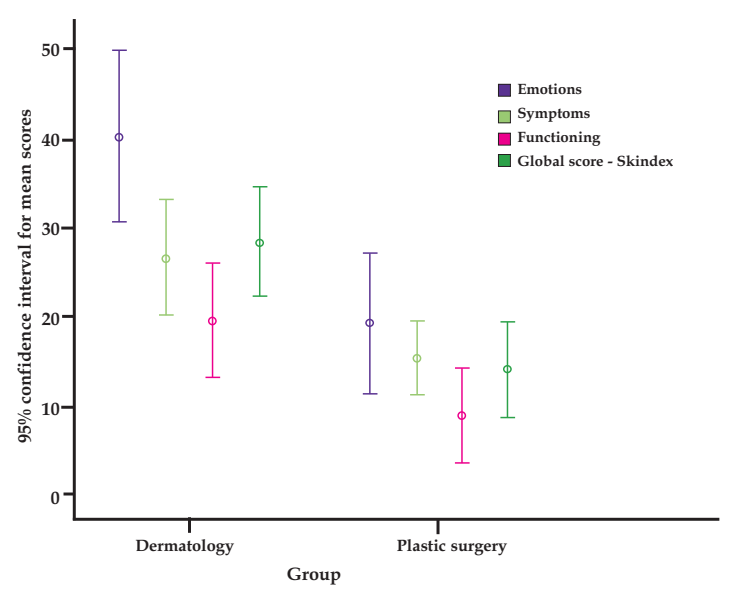

GrAPH 3: 95\% confidence interval of mean values for subscale and global Skindex-29 score per group. Total score - subscales

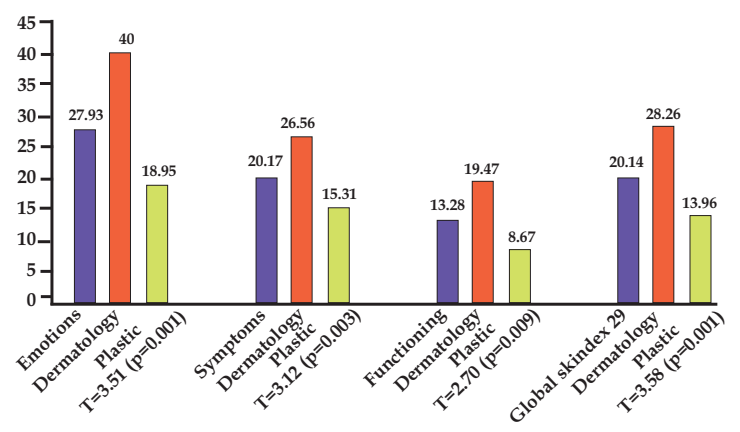

GrapH 4: Comparison of means values for global and subscale Skindex-29 scores per group 
TABLE 4: Correlation of the Skindex-29 and its subscales with the DLQI and the SF-36

\begin{tabular}{|c|c|c|c|c|}
\hline \multirow[t]{2}{*}{ Scores } & \multicolumn{4}{|l|}{ Spearman's correlation } \\
\hline & Total Skindex scores & Emotions & Symptoms & Functioning \\
\hline$\overline{\text { DLQI }}$ & $0.780^{* *}$ & $0.665^{\star *}$ & $0.622^{* *}$ & $0.803^{\star *}$ \\
\hline \multicolumn{5}{|l|}{ SF-36 } \\
\hline Mental health & $-0.569^{* *}$ & $-0.471^{* *}$ & $-0.327^{*}$ & $-0.597^{* *}$ \\
\hline Functional capacity & -0.18 & -0.021 & -0.151 & $-0.347^{*}$ \\
\hline Physical role limitation & -0.295 & -0.062 & -0.265 & $-0.497^{* *}$ \\
\hline Bodily pain & -0.226 & -0.13 & -0.035 & $-0.393^{* *}$ \\
\hline General health & $-0.400^{* *}$ & -0.259 & -0.255 & $-0.462^{* *}$ \\
\hline Vitality & $-0.514^{* *}$ & $-0.323^{*}$ & $-0.387^{*}$ & $-0.622^{* *}$ \\
\hline Social functioning & $-0.311^{*}$ & -0.259 & -0.251 & $-0.374^{*}$ \\
\hline $\begin{array}{l}\text { Limitation due to emotional aspects } \\
{ }^{*} \mathrm{p}<0.05 .{ }^{*} \mathrm{p}<0.01 \text {. }\end{array}$ & -0.26 & -0.169 & -0.121 & -0.249 \\
\hline \multicolumn{5}{|c|}{ Skindex Emotions and Functioning: $N=75$ patients. Skindex Symptoms and total score: $N=74$} \\
\hline \multicolumn{5}{|l|}{ DLQI: N=75 patients. } \\
\hline \multicolumn{5}{|c|}{ SF-36: $N=43$ (except for Emotional role limitation: $N=41$ ). } \\
\hline
\end{tabular}

tant in the search for an acceptable and intelligible instrument for the general population. In the present study, the sentences translated into Portuguese did not need to be modified after the interviews with patients, which confirms the clarity and objectiveness of questionnaire items.

Results also showed that the instrument measures three essential dimensions of quality of life associated with skin conditions and that the removal of any of the items did not lead to significant differences in the measuring power of the instrument. The dimensions and aspects evaluated in the Skindex seemed to be similar in the different cultures in which it has already been validated.

The correlation patterns between Skindex-29 and DLQI confirm the convergent validation evidence of questionnaires specific for dermatology. A statistically significant relationship was found when comparing DLQI scores with global Skindex scores and with the scores for each of Skindex dimensions (Table 4). On the other hand, when the Skindex-29 was compared with the SF-36, these similarities were not so expressive. There was a significant correlation only between the functional domain of the Skindex-29 with all domains of the SF-36 (except for emotional role limitation). Similar results were obtained in another study that compared the 61-item version of the Skindex with the SF-36. ${ }^{8}$ This study suggested that the Skindex-29 would theoretically assess some aspects of skin diseases more specifically and more accurately than a general health-related quality of life questionnaire such as the SF-36. This fact is not surprising, since the Skindex includes items that specifically investigate the health effects caused by skin disease, which does not occur with the SF-36, a questionnaire that takes into account only general health dimensions. This ability of the Skindex of specifically assessing quality of life in patients with skin diseases is shown to be particularly important when a more sensitive instrument is required (e.g., when researchers aim to compare patients with different skin diseases, detect changes in the effect of a given dermatosis on the patient, or perform clinical trials and longitudinal studies). Similarly, it is important to bear in mind that, although the Skindex is specific for skin conditions, it is not specific for any particular disease. Some pathologies already have quality of life assessment instruments that have been developed and validated in certain cultures. When such a specific instrument is available and the investigation focuses on a specific skin disease, this instrument is preferable to the Skindex29 , although specific and generic questionnaires provide complementary, not redundant, information.

Additional studies using the measuring properties of the Brazilian Portuguese version of the Skindex-29 will be able to confirm the equivalence of the present translation. Continuing to adapt the questionnaire to other cultures and languages will allow for international comparisons and multicenter studies, as well as for a possible increase in the quality of life of patients with skin diseases. The present study was not appraised by the original authors up to now.

\section{CONCLUSION}

The Brazilian Portuguese version of the Skindex-29 showed significant levels of reproducibility and validity and thus may be used in Brazil as a quality of life questionnaire specific for patients with skin changes. $\square$ 


\section{REFERENCES}

1. Faust HB, Mirowski GW, Chuang TY, Lewis CW, Gonin R, Melfi C, et al. Outcomes research: an overview. J Am Acad Dermatol. 1997;36:999-1006.

2. Finlay AY. Quality of life measurement in dermatology: a practical guide. $\mathrm{Br} \mathrm{J}$ Dermatol. 1997;136:305-14.

3. Ciconelli RM, Ferraz MB, Santos W, Meinão I, Quaresma MR. Brazilian-Portuguese version of the SF-36. A reliable and valid quality of life outcome measure. Rev Bras Reumatol. 1999;39:143-50.

4. Dini GM, Ferreira LM, Quaresma MR. Translation into Portuguese, cultural adaptation and validation of the ROSENBERG Self-esteem scale. Rev Soc Bras Cir Plást. 1997; 19:41-52.

5. Ferraz LB, Almeida FA, Vasconcellos MR, Faccina AS, Ciconelli RM, Ferraz MB. The impact of lúpus erythematosus cutaneous on the Quality of life: The BrazilianPortuguese version of DLQI. Qual Life Res. 2006;15:565-70.

6. Salek MS. Measuring the quality of life patients with skin disease. In: Walker SR, Rosser RM, editors. Quality of life assessment: Key Issues in the 1990s. Lancaster, UK: Kluwer Academic Publishers; 1993. p. 355-370.

7. Chren MM, Lasek RJ, Quinn LM, Mostow EN, Zyzanski SJ. Skindex, a quality-of-life measure for patients with skin disease: reliability, validity, and responsiveness. $J$ Invest Dermatol. 1996;107:707-13.

8. Chren MM, Lasek RJ, Quinn LM, Covinsky KE. Convergent and discriminant validity of a generic and a desease-specific instrument to meausure quality of life in patients with skin desease. J Invest Dermatol. 1997;108:103-7.

9. Guillemin F, Bombardier C, Beaton D. Cross-cultural adaptation of health-related quality of life measures: literature review and proposed guidelines. J Clin Epidemiol. 1993:46:1417-32.

10. Augustin M, Zschocke I, Seidenglanz K, Lange S, Schiffer A, Amon U: Validation and clinical results of the FLQA-d, a quality of life questionnaire for patients with chronic skin diseases. Dermatol Psychosom. 2000;1:12-7.

11. Amaral CSF, March MFBP, Sant'Anna CC. Quality of life in children and teenagers with atopic dermatitis. An Bras Dermatol. 2012;87:717-23

12. Torres RAT. Magalhães RF. Morcillo AM. Silva AS. Velho PENF. Comparison of quality of life questionnaires and their correlation with the clinical course of patients with psoriasis. An Bras Dermatol. 2011;86:45-9.

13. Costa MD, Terra FS, Costa RD, Lyon S, Dias-Costa AMD, Antunes CMF. Assessment of quality of life of patients with leprosy reactional states treated in a dermatology reference center. An Bras Dermatol. 2012;87:26-35.

14. Bottene IMC, Reis VMS. Quality of life of patients with paucibacillary leprosy. An Bras Dermatol. 2012;87:408-11.

15. Niemeier V, Kupfer J, Demmelbauer-Ebner M, Stangier U, Effendy I, Gieler U. Coping with acne vulgaris: Evaluation of the chronic skin disorders questionnaire in patients with acne. Dermatology. 1998;196:108-15.

16. Fernandes PM, Oliveira Filho RS, Ferreira LM. Auto-estima em pacientes com carcinomas de pele. Rev Col Bras Cir. 2007;34:361-6.

17. Barbato MT, Bakos L, Bakos RM, Prieb R, Andrade CD. Predictors of quality of life in patients with skin melanoma at the dermatology department of the Porto Alegre Teaching Hospital. An Bras Dermatol. 2011;86:249-56.

18. Lubeck DP: Measuring health-related quality of life in onychomycosis. J Am Acad Dermatol. 1998;38:S64-8.

19. Faria E, Blanes L, Hochman BS, Mesquita Filho M, Ferreira LM. Health-related quality of life, self-esteem, and function status of patients with leg ulcers. Wounds. 2011;23:4-10.

20. Neto MS, Abla LE, Lemos AL, Garcia ÉB, Enout MJ, Cabral NC, et al. The Impact of Surgical Treatment on the Self-Esteem of Patients with Breast Hypertrophy, Hypomastia, or Breast Asymmetry. Aesthetic Plast Surg. 2012;36:223-5.

21. Davanço RA, Sabino Neto M, Garcia EB, Matsuoka PK, Huijsmans JP, Ferreira LM Quality of life in the surgical treatment of gynecomastia. Aesthetic Plast Surg. 2009;33:514-7.

22. Oliveira ACS, Sabino Neto M, Veiga D F, Archangelo SCV, Andrade CHV, Novo NF, Ferreira LM . Mastectomia: Impacto na auto-estima das pacientes. Rev Bras Mastologia. 2006; 4:156-60.
23. de Lima Ramos S, Hochman B, Gomes HC, Abla LE, Veiga DF, Juliano Y, et al. Effect of nasal deviation on quality of life. Plast Reconstr Surg. 2011;128:132-6.

24. Alves MC, Abla LE, Santos Rde A, Ferreira LM. Quality of Life and Self-Esteem Outcomes Following Rhytidoplasty. Ann Plast Surg. 2005;54:511-4.

25. de Brito MJ, Nahas FX, Barbosa MV, Dini GM, Kimura AK, Farah AB, et al. Abdominoplasty and its effects on body image, selft-esteem, and mental health. Ann Plast Surg. 2010;65:5-10.

26. Abeni D, Picardi A, Pasquini P, Melchi CF, Chren MM. Further evidence of the validity and reliability of the Skindex-29: An Italian study on 2242 dermatological outpatients. Dermatology. 2002;204:43-9.

27. Chren MM, Lasek RJ, Flocke SA, Zyzanski SJ. Improved discriminative and evaluative capability of a refined version of Skindex, a quality-of-life instrument for patients with skin diseases. Arch Dermatol. 1997;133:1433-40.

28. Gill TM, Feinstein AR: A critical appraisal of the quality-of-life measurements. JAMA. 1994;272:619-26.

29. Spitzer WO. State ok science 1986: Quality of life and functional status as target variables for research. J Chronic Dis. 1987;40:465-71.

30. Jones-Caballero M, Peñas PF, García-Díez A, Badía X, Chren MM. The Spanish version of Skindex-29. Int J Dermatol. 2000;39:907-12.

31. Leplège A, Ecosse E, Zeller J, Revuz J, Wolkenstein P. Version Francaise du Skindex (Skindex - France): Adaptation et evaluation des proprietes psychometriques. Ann Dermatol Venereol. 2003;130:177-83.

32. Aksu AE, Urer MS, Sabuncu I, Saracoglu ZN, Chren MM.. Turkish version of Skindex29. Int J Dermatol. 2007:46:350-5.

33. Finlay AY, Khan GK. Dermatology Life Quality Index (DLQI) - A simple practical measure for routine clinical use. Clin Exp Dermatol. 1994;19:210-6.

34. Ware JE, Snow KK, Kosinski M, Gandek B. SF-36 health survey: manual and interpretation guide. Boston: New England Medical Center; 1993

35. Hunt SM, Alonso J, Bucquet D, Niero M, Wiklund I, McKenna S. Cross-cultural adaptation of health measures. Health Policy. 1991;19:33-44.
MAILING ADDRESS:
Henrique Ribeiro de Paula
Rua Napoleão de Barros, 715
Vila Clementino
04024-002 - São Paulo - SP
Brazil
E-mail: henriqueribeirodepaula@gmail.com

How to cite this article: Paula HR, Haddad A, Weiss MA, Dini GM, Ferreira LM. Translation, cultural adaptation and validation of the American quality of life index Skindex-29. An Bras Dermatol. 2014;89(4):600-7. 